\title{
MMP-9 Inhibition: a Therapeutic Strategy in Ischemic Stroke
}

\author{
Mayank Chaturvedi • Leszek Kaczmarek
}

Received: 22 July 2013 / Accepted: 15 August 2013 /Published online: 12 September 2013

(C) The Author(s) 2013. This article is published with open access at Springerlink.com

\begin{abstract}
Ischemic stroke is a leading cause of disability worldwide. In cerebral ischemia there is an enhanced expression of matrix metallo-proteinase-9 (MMP-9), which has been associated with various complications including excitotoxicity, neuronal damage, apoptosis, blood-brain barrier (BBB) opening leading to cerebral edema, and hemorrhagic transformation. Moreover, the tissue plasminogen activator (tPA), which is the only US-FDA approved treatment of ischemic stroke, has a brief 3 to $4 \mathrm{~h}$ time window and it has been proposed that detrimental effects of tPA beyond the $3 \mathrm{~h}$ since the onset of stroke are derived from its ability to activate MMP-9 that in turn contributes to the breakdown of BBB. Therefore, the available literature suggests that MMP-9 inhibition can be of therapeutic importance in ischemic stroke. Hence, combination therapies of MMP-9 inhibitor along with IPA can be beneficial in ischemic stroke. In this review we will discuss the current status of various strategies which have shown neuroprotection and extension of thrombolytic window by directly or indirectly inhibiting MMP-9 activity. In the introductory part of the review, we briefly provide an overview on ischemic stroke, commonly used models of ischemic stroke and a role of MMP9 in ischemia. In next part, the literature is organized as various approaches which have proven neuroprotective effects through direct or indirect decrease in MMP-9 activity, namely, using biotherapeutics, involving MMP-9 gene inhibition using viral vectors; using endogenous inhibitor of MMP-9, repurposing of old drugs such as minocycline, new chemical entities like DPb99, and finally other approaches like therapeutic hypothermia.
\end{abstract}

Keywords Ischemic stroke $\cdot$ MMP-9 $\cdot$ Neuronal damage MMP inhibitors · TIMP-1 - Therapeutic target .

Neuroprotection

M. Chaturvedi $(\bowtie) \cdot$ L. Kaczmarek $(\bowtie)$

Laboratory of Neurobiology, Nencki Institute, Pasteura 3, 02-093 Warsaw, Poland

e-mail: m.chaturvedi@nencki.gov.pl

e-mail: 1.kaczmarek@nencki.gov.pl

\author{
Abbreviations \\ MMP-9 Matrix metalloproteinases-9 \\ TIMP-1 Tissue Inhibitor of Matrix Metalloproteinases-1 \\ tPA tissue-type plasminogen activator \\ BBB Blood Brain Barrier
}

\section{Introduction}

Ischemic Stroke

Causes, Classification, and Management

Worldwide, stroke is among the leading causes of death and serious disability [1]. In stroke, there is a disruption in the blood supply to the brain leading to rapid loss of brain function. As a result, the affected area of the brain cannot function, which might result in an inability to move one or more limbs on one side of the body, inability to understand or speak, or to see one side of the visual field.

Strokes are classified as ischemic and hemorrhagic. Ischemic strokes are caused by obstruction of the blood supply, while hemorrhagic strokes results from rupture of a blood vessel. Most of the strokes (about 85-90 \%) are ischemic. Occasionally, hemorrhagic strokes develop in ischemic brain, and called as hemorrhagic transformation of ischemic stroke, although it is unknown how many hemorrhages actually start off as ischemic stroke.

Currently, there is only one US-FDA approved treatment for ischemic stroke, i.e., tissue-type plasminogen activator (tPA). To be effective, tPA must be administered intravenously within the first $3-4 \mathrm{~h}$ of the event due to risks of hemorrhagic transformation of ischemic stroke [2-4]. Because of narrow time window, only a few percent of patients qualify for this treatment. Hence there is a need to find new therapeutic targets for increasing the therapeutic time window of tPA and protect neurons from ischemic injury. 


\section{Models for Ischemic Stroke}

To test potential therapeutic interventions and to understand underlying mechanisms in ischemic stroke there are several in vitro and in vivo models available in different species. Therefore, depending on the research questions a specific model is chosen. In this section, we will give a brief outline of the most commonly used models of ischemic stroke rather than their precise methodology, which is beyond the scope of this review and has been described in detail by others [5-8].

In Vitro Models There are several in vitro models available for ischemic stroke, but the most established and widely used in vitro model for ischemia is oxygen glucose deprivation (OGD). In this model primary neuronal cultures, organotypic cultures, and acute brain slices can be incubated in deoxygenated, glucose-free medium to mimic the interruption of the supply of oxygen and nutrients to the brain parenchyma [9].

An alternative to OGD is chemical ischemia model, which can simulate certain aspects of ischemic brain injury. In this model, sodium azide or sodium cyanide, inhibitors of oxidative metabolism, often together with 2-deoxyglucose, an inhibitor of glycolysis, are used to induce hypoxia and hypoglycemia in cultures [10].

In Vivo Models Rodents are the most commonly used animals for ischemic stroke model because of a resemblance to humans in their cerebral anatomy and physiology, small size, and low cost. There are varieties of rat and mouse models available for cerebral ischemia; see reviews $[5,7,8,11]$. The most basic models of mimicking complete global brain ischemia are: decapitation, aorta/vena cava occlusion by coagulation of both vertebral arteries, followed by the transient occlusion of both common carotid arteries (four-vessel occlusion); external neck tourniquet or cuff and cardiac arrest. For incomplete global ischemia, the most commonly used model is hypoxic ischemia. In this model there is permanent unilateral carotid artery ligation with a subsequent 3 -h exposure to a hypoxic environment ( $8 \%$ oxygen) that creates a unilateral infarct in the hemisphere ipsilateral to the ligation. The area of injury is typically concentrated in periventricular regions of the brain, especially cortical and hippocampal regions [12].

Experimental focal cerebral ischemia is the most powerful model of ischemic stroke because it closely mimic the changes that occur during and after human ischemic stroke; see review [13]. There are various approaches for inducing focal cerebral ischemia, for instance thromboembolic stroke is produced by blood clot formation. This model is of great interest because it is closest in resemblance to human ischemic stroke and its utility in evaluating thrombolytic therapies. Another model called photo-thrombotic stroke is a way of inducing thrombosis by systemic injection of a photoactive dye in combination with illumination by a light beam transmitted through the intact skull. The illumination of photo dye causes alteration in dye which leads to oxidative damage to the endothelium leading to platelet aggregation. Microsphere embolization is another model, with the severity of ischemic damage related to the number of emboli used. Middle cerebral artery occlusion (MCAO) is surgical occlusion using a filament.

It shall, however, be stressed that since human stroke comes in many forms, no single animal model is able to encompass all of the variables known to affect human ischemic stroke. Thus none of the available models perfectly simulates human stroke which makes it very difficult to interpret the results in context of human stroke. Hence, this is one of the main reasons of many drugs failing in clinical trials, because of poor translation of preclinical data to clinical stage. Therefore, preclinical data from animal models should be very cautiously interpreted before extrapolating to humans.

\section{Pathological Assessment of Brain Damage in Ischemic Models}

For assessing neuronal damage in in vitro models, propidium iodide is most commonly used. It is a marker for dead or dying cells, as it only enters and stains cell nuclei after loss of cell membrane integrity. Other colorimetric assays for assessing cytotoxicity after ischemia include, 3-(4, 5-dimethyl-2thiazolyl)-2, 5-diphenyl-2H-tetrazolium bromide (MTT) assay and lactate dehydrogenase (LDH) assay. MTT is reduced by metabolically active cells to insoluble purple formazan dye crystals which can be visualized by colorimetry. While LDH is an enzyme which converts pyruvic acid to lactic acid and it can be readily detected when cell membranes are no longer intact.

The effectiveness of in vivo models can be estimated by measuring tissue loss and functional deficits. Infarct volume is one of the major criteria which is traditionally measured by quantitative histology. For infarct size measurement, tripenthyltetrazolium chloride (TTC) and hematoxylin-eosin (H\&E) staining is most commonly used. TTC is a redox indicator which indicates cellular respiration. After TTC staining the areas of potential damage after ischemic stroke are paler as compared to healthy viable tissue, which will stain deep red after stroke.

Blood brain barrier (BBB) integrity is another important parameter which is most commonly assessed by Evans blue test. Evans blue is a marker of BBB permeability for albumin. There are also various other ways for measuring BBB permeability and one of the most common method after Evans blue test is by observing immunolocalization of IgG. It has been shown that there is increased permeability to endogenous proteins such as $\operatorname{IgG}$ when BBB is compromised and hence this property of $\operatorname{IgG}$ is capitalized to measure $\mathrm{BBB}$ integrity. Further, immuno-cyto/histo-chemistry for tight junction protein such as ZO-1, occludin, and claudin-5 is also a good indicator of BBB integrity. 
Apart from these morphological parameters, neurological assessments are very useful but these deficits are most difficult to assess in animals. Usually, motor deficits are quantified by using sensorimotor tests including rotarod, grid walking, limb placing, beam walking, and sticky label test [13].

MMP-9 and Its Role in Ischemia

\section{Physiological and Pathological Significance of MMP-9}

Matrix metalloproteinases (MMPs) form a mutligene family of zinc-dependent endopeptidases of over 25 enzymes, mostly secreted and acting outside the cells. Their targets include other proteinases, proteinase inhibitors, clotting factors, chemotactic molecules, latent growth factors, growth factorbinding proteins, cell surface receptors, cell-cell adhesion molecules, and virtually all structural extra-cellular matrix proteins. Thus MMPs are able to regulate many biologic processes [14].

In particular, MMP-9 (gelatinase B, $92 \mathrm{kDa}$ type IV collagenase) is produced in a latent form in the cell and after release to extracellular space, it is activated by cleavage off the propeptide [15]. It is involved in the breakdown of extracellular matrix in various physiological processes, such as embryonic development, reproduction, angiogenesis, bone development, wound healing, cell migration, and recently, it has been shown to be increasingly important in several aspects of central nervous system activity including its role in learning and memory $[16,17]$.

Enhanced expression and activities of MMPs especially MMP-9 have been observed under numerous pathologic conditions $[16,18]$, including many neuropsychiatric disorders [19] such as epilepsy, autism spectrum disorders, addiction, and bipolar disorders and schizophrenia. It has also been implicated in other CNS disorders such as Alzheimer's disease, multiple sclerosis, brain tumors, Guillain-Barre syndrome, spinal cord injury, ischemic stroke, and excitotoxic/ neuroinflammatory processes $[16,20]$.

\section{Current Techniques for Assessing MMP-9 Activity}

For assessing MMP-9 activity, the most commonly used technique is zymography and reverse zymography for assessing activity of its inhibitors. There are variations of this method such as in situ zymography and real-time zymography. Standard MMP-9 zymography is a variation on acrylamide gel electrophoresis. The gel used for zymography, commonly referred to as zymogram, contains gelatin (substrate of MMP9) incorporated directly into the gel during polymerization. Following electrophoresis of the sample containing MMP-9 (gelatinase B), SDS is removed from the gel by exchange with Triton X-100. This allows the gelatinases in the sample to renature and auto-activate, following incubation of gels in activation buffer. MMP-9 activity is revealed by coomassie blue staining. A variation of standard zymography called as real-time zymography in which fluorescently tagged (FITCtagged) gelatin which allows real-time monitoring of activity under UV light and no coomassie blue staining is needed.

For assessing MMP-9 activity in histological sections in situ zymography is used. In this method frozen sections are placed on glass slides coated with fluorescently labeled matrix proteins. After incubation MMP activity can be observed as black patches in the fluorescent background due to proteolysis of the matrix protein [21]. In another variation, frozen sections are incubated with dye-quenched (DQ) gelatin which upon proteolysis by MMP-9 becomes fluorescent. Hence, the MMP-9 activity can be visualized in high resolution as bright fluorescence spots [22].

Another method for measuring MMP-9 activity is gelatinase assay. In this method DQ gelatin is used as a substrate for MMP-9. As DQ gelatin is cleaved by MMP-9, it has fluorescence which can be quantified by microplate reader.

\section{Increase in MMP-9 Activity After Cerebral Ischemia}

During the last decade, a vast clinical data have been accumulated from many studies which confirm critical role of MMP-9 and its deleterious effects during human stroke and reperfusion injury [23-27]. These results have also been correlated in number of animal models, which have shown a marked increase of MMP-9 expression in ischemic stroke [28-32].

MMP-9 might be released in response to ischemic insult from neurons, oligodendroglia, reactive astrocytes, and activated microglia [33]; furthermore, free radicals, tPA, and other molecules which are released after ischemic insult have the capacity to activate MMP-9; see reviews [34, 35]. This increase in MMP-9 is further associated with various complication including excitotoxicity, neuronal damage [36], apoptosis [37, 38], oxidative stress [39], interference with oxidative DNA repair mechanisms [40], and most importantly BBB opening leading to cerebral edema and hemorrhagic transformation [41] after cerebral ischemia. Moreover, it has been proposed that detrimental effects of tPA beyond the $3 \mathrm{~h}$ of stroke onset are derived from tPA's ability to activate MMP-9 [42-44]. This in turn contributes to the breakdown of BBB [44-47].

The first study showing increase of MMP-9 in human brain after ischemia was probably done by Clark et al. [48]. They showed that MMP-9 activity was markedly elevated in the infarcted human cerebral tissue after 2 days post-infarction as compared to non-infarcted tissue. This was followed by Heo et al. who showed, using gelatin zymography that after MCAO in non-human primates, only MMP-9 increases transiently after $2 \mathrm{~h}$ of stroke onset not MMP-2 [49] Fig. 1. They showed that MMP-9 expression only was significantly increased in subjects with hemorrhagic transformation and not any other MMP. These results were corroborated in many other stroke models also, such as mouse model of focal cerebral ischemia, where it has been shown that MMP-9 is 


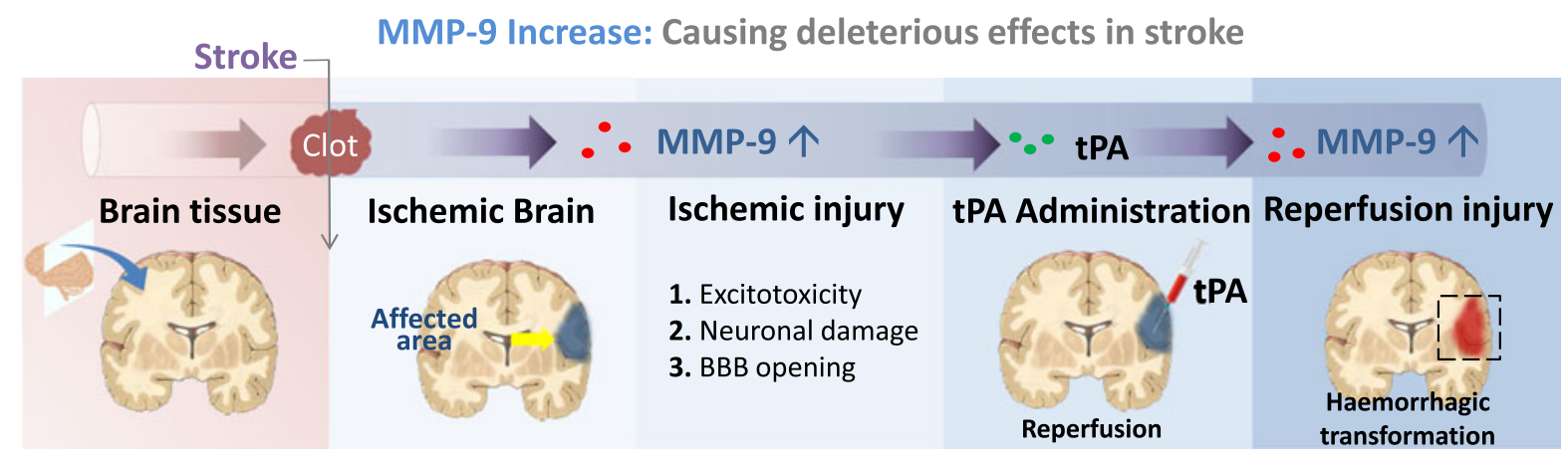

"Combination therapy of MMP-9 inhibitor with tPA can be Neuroprotective"

MMP-9 inhibition: Strategies with proven decrease in MMP-9 activity

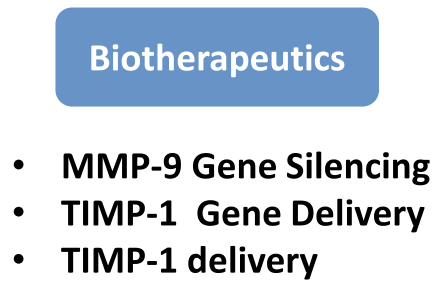

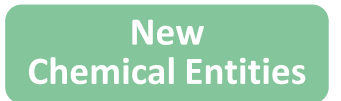

- DP-b99

- SB-3CT
Miscellaneous

\section{- Platinum Nanoparticles \\ - Therapeutic Hypothermia}

Fig. 1 Obstruction of blood supply causes ischemic injury which in turn causes activation of various factors including MMP-9, which is suggested to be involved in excitotoxicity, neuronal damage and BBB opening. Reperfusion, using TPA, causes further activation of MMP-9 which has been shown to be involved in hemorrhagic transformation of ischemic

increased after onset of stroke and further these increase correlates with increase in BBB permeability which was determined by Evans blue test $[50,51]$. Similarly, there are multiple data from other animal models of ischemia, which suggest increase in MMP-9 relating to hemorrhagic transformation [52]. Furthermore, recent data confirm the presence of high MMP-9 levels not only in infarct tissue but also in the peri-infarct areas, suggesting that MMP-9 involvement in the process of infarct size growth [53-55]. Therefore, it has been suggested that MMP-9 can be considered as possible marker for brain ischemia, as well as a predictor of hemorrhage in patients treated with tPA [56].

These results have collectively showed excessive activation of MMP-9 has deleterious effects to the brain, which are associated with BBB leakage and inflammation. Thus, inhibition of MMP-9 is considered as a potential therapeutic target.

\section{MMP-9 Transgenic and Knock Out in Ischemic Stroke}

The first study on MMP-9 knock out (KO) mice was from Lo's group in traumatic brain injury model which showed that MMP-9 KO mice have less motor deficits than wild-type (WT) mice [57]. In focal cerebral ischemia model MMP-9 KO mice have shown significant BBB protection. The BBB integrity by Evans Blue test has been shown to be significantly

stroke and also responsible for brief therapeutic window of tPA. Therefore, it has been proposed that combination therapy of MMP-9 inhibitor with tPA can be neuroprotective. In this review, we discuss various approaches of MMP-9 inhibition which have shown to be therapeutically beneficial

attenuated in MMP-9 KO mice as compared with WT [58]. Furthermore, in white matter, degradation of the MMP-9 possible substrate myelin basic protein was also significantly reduced in KO mice as compared with WT [58]. Another study on cerebral hypoxic-ischemia model in immature brain on post-natal 9-day-old mice demonstrated that MMP-9 KOs are protected from ischemic damage [59]. Apart from MMP-9 KO mice, recently, CEACAM-1 (carcino-embryonic antigenrelated cell adhesion molecule-1) KO mice, has also been shown to protect BBB breakdown, after tMCAO in mouse; through MMP-9-inhibition-mediated pathway [60]. Taken together, it has been shown by different ischemia models that MMP-9 KO mice (and also others KO such as CEACAM1 $\mathrm{KO}$ ) have reduced ischemic brain damage.

\section{TIMP-1 Transgenic and KO in Ischemic Stroke}

Tissue inhibitor of matrix metalloproteinases 1 (TIMP-1) is known to bind MMP-9 with high affinity and to block its enzymatic function. TIMP-1 is upregulated following an excitotoxic injury, which has been hypothesized to be part of a general neuronal response that possibly mediates tissue reorganization and/or neuroprotection [61]. Being endogenous inhibitor of MMP-9 it is suggested that TIMP-1 can play a neuroprotective role in cerebral ischemia. Therefore to 
evaluate neuroprotection by genetic inhibition of MMP-9 there were various studies using TIMP-1 transgenic mice.

It has been shown that in TIMP-1 overexpressing transgenic mice after $2 \mathrm{~h}$ of transient focal cerebral ischemia, MMP-9 levels were lower as compared with WT [62]. Correspondingly, BBB leakage is also ameliorated by TIMP-1 overexpression, and after $24 \mathrm{~h}$ infarction volumes they have reduced. On the other hand, in TIMP-1 KO mice, MMP-9 protein expression and gelatinolytic activity were significantly more augmented after cerebral ischemia than those in WT mice. This MMP-9 activity was also accompanied by exacerbated BBB disruption, neuronal apoptosis, and ischemic injury [63]. Taken together, TIMP-1 transgenic and $\mathrm{KO}$ mice provide a first proof-of-principle that genetic MMP9 inhibition may be neuroprotective after ischemic brain injury.

In the next section, we will discuss the current status of various pharmacological approaches for protecting ischemic brain from deleterious effects of MMP-9. There have been attempts by using endogenous and chemical MMP-9 inhibitors in combination with tPA to protect the ischemic brain and further increasing thrombolytic window of tPA.

\section{Therapeutics Approaches of MMP-9 Inhibitions}

During the last two decades, a great deal of attention has been focused on neuroprotective therapies [64]. MMP-9 has been identified as aberrantly overactive in ischemia, potentially causing deleterious effects during ischemia and after reperfusion, and hence its inhibition has been considered as a potential therapeutic target [65]. The idea of neuroprotection by inhibiting MMP-9 in ischemic stroke is not very old, but multiple studies have been done during the last decade using various strategies for MMP-9 inhibition. In a number of in vitro and in vivo models, MMP-9 inhibition has shown to be neuroprotective, e.g., by using lentiviral-mediated MMP-9 gene silencing [66] or by delivering recombinant TIMP-1 in native form $[67,68]$ or by PLGA nanoparticles [69]. Notably, minocycline appears to be the most studied drug for ischemic stroke.

\section{Biotherapeutics}

Biotherapeutics are biological materials such as recombinant proteins, monoclonal antibodies, and siRNAs used to encompass therapeutics effects. Several biotherapeutic approaches are being used to inhibit MMP-9, such as MMP-9 neutralizing antibodies, MMP-9 siRNA, or shRNA delivered through adenoviral vectors, lentiviral vectors, quantum dots, and gold nanoparticles, which all have ameliorated deleterious consequences of ischemic stroke. Furthermore, there have been studies of inhibiting MMP-9 activity by administering MMP-9 neutralizing monoclonal antibody even after focal ischemic insult that have shown to decrease the infarct size [70].

\section{MMP-9 Gene Silencing}

Various studies on focal cerebral ischemic models by MMP-9 gene silencing through adenoviral and lentiviral vectors have shown neuroprotective effects. There was significant reduction in brain infarction volume, brain water content, and neurobehavioral deficits following lentiviral-deliverymediated MMP-9 silencing by LV-mmp9shRNA treatment [66]. Additionally, Evan's blue and IgG extravasation were reduced showing BBB integrity. Another study, showing therapeutic application of MMP-9 gene silencing by intracerebroventricular injection of liposomal formulation containing MMP-9 siRNA within $60 \mathrm{~min}$ after $2 \mathrm{~h}$ of a middle cerebral artery occlusion-induced focal ischemia rat model showed BBB protection [71]. These findings provide evidence that a liposomal formulation of siRNA might be used in vivo to silence the MMP-9 gene and could potentially serve as an important therapeutic alternative in patients with cerebral ischemia.

Also, there have been novel strategies of MMP-9 gene silencing such as by gold nano rods (GNRs), which can electrostatically bind with MMP-9 siRNA to form a nano complex (called as nanoplex). This nanoplex can be readily up taken by brain microvascular endothelial cells (BMVECs) that constitute the BBB. It has been shown that silencing of MMP-9 gene expression by this GNR nanoplex in BMVEC increases the expression of tight junction proteins namely $\mathrm{ZO}$ 1, Occludin, and Claudin-5 thereby decreasing endothelial permeability [72].

There are other novel studies involving application of a nanoparticle-based siRNA delivery system, such as nanoplex of quantum dot (QD) and MMP-9-siRNA, which has been shown to silence MMP-9 gene expression in BMVECs and other cells, e.g., leukocytes [73]. Their study showed that this QD nanoplex downregulated the expression of MMP-9 gene in BMVEC and prevented breakdown of the BBB and thus inhibited subsequent invasion of the CNS by infected and inflammatory cells. The results also showed that silencing of MMP-9 gene expression resulted in the upregulation of such ECM proteins as collagen I, IV, V, and a decrease in endothelial permeability, which was measured by trans-endothelial resistance across the $\mathrm{BBB}$ in the in vitro $\mathrm{BBB}$ model. Furthermore, they have shown that MMP-9 gene silencing results in an increase in expression of the gene TIMP-1 which strengthen the hypothesis of TIMP-1's role in neuroprotection.

\section{TIMP-1 as Therapeutic Strategy}

In aggregate, the literature indicates the importance of a balance between the levels of MMP-9 and its natural inhibitor TIMP-1 in maintaining the basement membrane integrity in ischemic stroke, as proteolysis of MMP-9 is tightly controlled 
by TIMP-1. Therefore, TIMP-1 is considered as a therapeutic strategy for ameliorating deleterious effects of MMP-9 in ischemic stroke.

Notably, it has been demonstrated earlier that within hours of applying recombinant TIMP-1 either in native form or through adenoviral-mediated gene transfer to organotypic hippocampal cultures, neurons are highly protected against excitotoxic injury induced by kainic acid [68].

In vivo delivery of TIMP-1 by using adenoviral vectors [74] has been shown to significantly reduce neuronal damage (by approximately $50 \%$ ) after TIMP-1 gene transfer, as compared to control. The only main obstacle of using TIMP-1 as a therapeutic agent is it has short half-life in vivo [75, 76], therefore, there have been attempts to extend its bioavailability such as PEGylating the TIMP-1 [76], so in future it can be developed as a neuroprotective. Furthermore, TIMP-1 being large protein cannot cross the BBB. Therefore, to enhance it BBB penetration and increase its circulation time, TIMP-1loaded PLGA nanoparticles have been formulated [69]. These nanoparticles have shown in vitro neuroprotection-mediated MMP-9 inhibition [69]. It is suggested that neuroprotective effects of TIMP-1 may arise not only due to MMP-9 inhibition but also in combination of several other mechanisms, such as eythropoietin-induced neuroprotection as shown in oxygen glucose deprivation model by [67].

\section{Re-purposing Old Drugs}

As drug pipeline of pharmaceutical industries are drying up, the drug repositioning or repurposing has thus grown into importance during the last decade $[77,78]$. The tetracycline derivative, minocycline which is a broad-spectrum antibiotic is the most studied drug for its neuroprotective function in ischemic stroke. Remarkably, all studies have shown the decrease in MMP-9 activity after minocycline treatment and subsequent neuroprotection. There are a few reasons why minocycline is chosen a lead molecule for exploring its neuroprotective effects. First, it has proven safety record over decades as an antibiotic. Secondly, it has very good ability to diffuse into the central nervous system at clinically effective levels. And finally, aside from its antimicrobial properties, minocycline has been found to have inhibitory effects on matrix metalloproteinases $[79,80]$. This suggests that it may have potential for development into an effective treatment for ischemic stroke [81-83]. Concordantly, minocycline has been found to have neuroprotective effects in animal models of a number of diseases including stroke [84].

There are many pieces of evidence derived from in vitro and in vivo studies that neuroprotection mediated by minocycline is through MMP-9 inhibition. In vitro it has been shown that minocycline ameliorates oxygen-glucose deprivation-induced cell cytotoxicity and down-regulate the production and activity of matrix metalloproteinase-9 [85]. In vivo it has been shown that minocycline inhibits enzymatic activity of gelatin proteases activated by ischemia after experimental stroke and is likely to be selective for MMP-9 at low doses [81]. Furthermore, in vivo studies have shown that the neuroprotective effects of minocycline may be mediated by interfering with MMPs. Koistinaho et al. has compared minocycline's protective effect in WT and MMP-9 KO mice [86]. They showed that minocycline inhibited ischemiaprovoked pro-MMP-9 induction in WT mice, but was not protective in MMP-9 KO mice.

Combinational therapy of minocycline with tPA has shown to extend the $3 \mathrm{~h}$ window to $6 \mathrm{~h}$ in embolic focal ischemia model in rat [87]. Such treatment results also in a decrease of MMP-9 plasma levels, reduced infarction, and ameliorated brain hemorrhage. Moreover, the exploratory clinical trials of minocycline have shown to improve neurological outcome in stroke and it was found that it lowers plasma matrix metalloproteinase-9 in tPA-treated subjects [88]. In the future, combining minocycline with tPA may prevent the adverse consequences of thrombolytic therapy through suppression of matrix metalloproteinase-9 activity [89].

Another class of drug, Statins which are 3-hydroxy-3methylglutaryl coenzyme A reductase inhibitors, have shown pleiotropic effects and are considered as potential neuroprotective agents in the treatment of stroke. Atorvastatin which is a member of statin family and notably marketed by Pfizer as a calcium salt under the trade name "Lipitor" which is the highest selling drug, used for lowering blood cholesterol and hence it has well-proven safety record. Atorvastatin has also been studied for extending thrombolytic window of tPA [90]. In rats after embolic middle cerebral artery occlusion, combination of atorvastatin and TPA have been shown to extended the therapeutic window for stroke to $6 \mathrm{~h}$ without increasing the incidence of hemorrhagic transformation. Furthermore, this combination therapy also abolished tPA-induced upregulation of MMP-9, as well as the degradation of collagen IV, leading to a reduction in the incidence of hemorrhagic transformation $(10 \%)$ as compared with the incidence during monotherapy of the tPA group (40\%).

Recently, melatonin, which is a highly potent free radical scavenger, has been shown to have neuroprotective effects by reducing the elevated matrix metalloproteinase- 9 level in a rat photothrombotic stroke model. These findings suggest that oxidative stress by free radicals might be highly associated with the activation of the MMP-9 pathway and that melatonin might protect against BBB disruption by reducing MMP-9 activity after cerebral ischemia through the reduction of oxidative stress by scavenging free oxygen radicals [91]. Another molecule, a lipoxin A(4) analog LXA(4) methyl ester (LXA(4)ME), also prevents BBB dysfunction in a rat model of focal cerebral ischemia reperfusion injury [92]. Its effects are attributed to reduction in MMP-9 activity which is mediated by increase in TIMP-1 expression. 
Hematopoietic growth factors such as erythropoietin (EPO) provides neuroprotection and promotes neurogenesis and angiogenesis after stroke, similarly thrombopoietin (TPO), which has significant homology with EPO (23\%) at the amino-acid level and with neurotrophins (e.g., the highest homology with BDNF, $36 \%$ ) in the $\mathrm{N}$ terminal region has shown efficacy in reducing ischemic brain injury in MCAO model [93]. It protects the brain from the early, negative effects on the BBB and improving functional outcome, partly by inhibiting the strokeinduced increase in MMP-9. Angiotensin type 1 receptor blocker such as olmesartan can reduce the reactive upregulation in brain angiotensin II, MMP-2, MMP-9, and membrane type 1-MMP in the ischemic area to improve stroke index score, infarct volume, and cerebral edema in cerebral ischemia model [94]. Non-steroidal anti-inflammatory drugs such as indomethacin inhibitor of COX-1 and COX-2 has shown to significantly reduce the expression and activity of MMP-9 as assessed by immunoblotting and gelatin-substrate zymography and attenuated the brain edema [95].

\section{New Chemical Entities}

DP-b99 is a new chemical entity that is discovered and developed by D-Pharm's proprietary Membrane Active Chelators (MAC) platform technology. DP-b99 has been shown to attenuate detrimental zinc-dependent membrane-associated processes, such as calpain activation and tumor necrosis factor- $\alpha-$ induced activation of matrix metalloproteinase-9 [96]. Another MMP inhibitor KB-R7785 showed protective effects against brain infarct formation in focal cerebral ischemia [97].

Another new molecule SB-3CT, which is presented as a highly selective inhibitor that is known to target MMP-9, has protected neurons from apoptosis in transient focal cerebral ischemia [98]. While on the other hand another study done on immature brain (21 old postnatal Wistar rats) showed that despite of significantly inhibiting MMP-9 activity after unilateral carotid artery occlusion unilateral carotid artery occlusion followed by exposure to hypoxia ( $8 \%$ oxygen for $1 \mathrm{~h}$ ), SB-3CT failed to confer significant neuroprotection in [99]. Hence, further investigations are still needed by using other recently reported selective water-soluble version of SB-3CT or another MMP-9 selective inhibitor to resolve the role of MMP-9 in the etiology of ischemic injury.

\section{Miscellaneous}

From the available data, it is clear that ischemic injury involves numerous pathways via which cerebral hence the therapeutic should be developed with the capacity to inhibit multiple mechanisms simultaneously to provide synergistic beneficial clinical effects for stroke patients. Therapeutic hypothermia is among the most promising technique as a post-ischemic pharmacological interventions and has been shown to provide long-lasting neuroprotection in preclinical studies [100, 101]. It has been shown that therapeutic hypothermia also decreases MMP-9 activity in rat after MCAO [102]. Moreover, combination of minocycline and hypothermia has shown to have additional neuroprotection with decrease in MMP-9 activity [103]. There have been important preclinical data suggesting that moderate hypothermia significantly decreases MMP-9 activity and hence protect the ischemic brain [104, 105]. Recently, Zhao and colleagues have shown that focal mild hypothermia (for $6 \mathrm{~h}, 33^{\circ} \mathrm{C}$ ) can decrease expression of MMP-9 and TIMP-1, resulting in improved neurological function [106]. Furthermore, in humans after acute ischemic stroke within $12 \mathrm{~h}$ of stroke onset when the patients were treated with moderate hypothermia, i.e., patients were kept at $33^{\circ} \mathrm{C}$ body-core temperature for 48 to $72 \mathrm{~h}$. It has been shown that MMP-9 levels are significantly lower in patients treated with moderate hypothermia as compared to the patients treated with tPA [25]. And conversely, laminin levels are significantly lower after tPA treatment as compared to hypothermia-treated patients, suggesting lowering of MMP-9 activity after hypothermia treatment. Therapeutic hypothermia has been investigated in clinical trials and despite a reasonable amount of clinical data still additional trials are needed to define the optimal time window, temperature regimen, and precise clinical indications for induction of therapeutic hypothermia in the setting of acute stroke [107].

There are various other approaches which do not directly inhibits MMP-9 but it has been shown that they do decrease MMP-9 activity significantly. For e.g. platinum nanoparticles as a novel reactive oxygen species scavenger were used after MCAO and examined for their neuroprotective effects. The study demonstrated that nanoparticles treatment ameliorates neurological function and brain damage in acute cerebral infarction with neuroprotective effect and most importantly the nanoparticles also reduced MMP-9 activity [108].

\section{Summary and Prospects}

To conclude, accumulating data suggest that MMP-9 concentration is significantly increased in both animal models of cerebral ischemia and in human stroke. This increased MMP-9 level is associated with neuronal damage, apoptosis, BBB opening followed by cerebral edema, hemorrhagic transformation, and reperfusion injury. Therefore MMP-9 is among the most important therapeutic targets in ischemic stroke. We suggest that, as MMP-9 structure and its regulations at gene level and protein level have been known, therefore, compounds which can directly inhibit its activity or decrease MMP-9 expression by blocking signal pathways should be exploited for developing new therapeutics. Further, the endogenous inhibitors of MMP-9, i.e., TIMP-1 should also be 
explored for its possible beneficial effects. In future, combination therapies of TPA with MMP-9 inhibitor may be useful for decreasing the risk and severity of thrombolytic therapy for human stroke.

Acknowledgments We wish to thank the Foundation of Polish Science for the grant no. MPD4-5071 funded by European regional development funds of European Union. LK was supported by the European Union's Seventh Framework Programme (FP7/2007-2013) under grant agreements no. 201024 and no. 202213 (European Stroke Network/ARISE).

Conflict of Interest The author declares no conflicts of interest that are directly relevant to the content of this review.

Open Access This article is distributed under the terms of the Creative Commons Attribution License which permits any use, distribution, and reproduction in any medium, provided the original author(s) and the source are credited.

\section{Reference}

1. Towfighi A, Saver JL (2011) Stroke declines from third to fourth leading cause of death in the United States historical perspective and challenges ahead. Stroke 42(8):2351-2355

2. Crunkhorn S (2008) Stroke: widening the therapeutic window? Nat Rev Drug Discov 7(8):643-643

3. The National Institute of Neurological Disorders and Stroke (1995) Tissue plasminogen activator for acute ischemic stroke rt-PA. Study Group 333(24):1581-1587

4. Tilley BC, Lyden PD, Brott TG, Lu M, Levine SR, Welch K (1997) Total quality improvement method for reduction of delays between emergency department admission and treatment of acute ischemic stroke. The National Institute of Neurological Disorders and Stroke rt-PA Stroke Study Group. Arch Neurol 54(12):1466-1474

5. Durukan A, Tatlisumak T (2008) Animal models of ischemic stroke. Handb Clin Neurol 92:43-66

6. Wang-Fischer Y (2008) Manual of stroke models in rats. CRC, New York

7. Graham SM, McCullough LD, Murphy SJ (2004) Animal models of ischemic stroke: balancing experimental aims and animal care. Comp Med 54(5):486-496

8. Wiebers D, Adams H Jr, Whisnant J (1990) Animal models of stroke: are they relevant to human disease. Stroke 21(1):1-3

9. Cimarosti H, Henley JM (2008) Investigating the mechanisms underlying neuronal death in ischemia using in vitro oxygenglucose deprivation: potential involvement of protein SUMOylation. Neuroscientist 14(6):626-636

10. Kume T, Nishikawa H, Taguchi R, Hashino A, Katsuki H, Kaneko S, Minami M, Satoh M, Akaike A (2002) Antagonism of NMDA receptors by $\sigma$ receptor ligands attenuates chemical ischemiainduced neuronal death in vitro. Eur J Pharmacol 455(2):91-100

11. Howells DW, Porritt MJ, Rewell SS, O'Collins V, Sena ES, van der Worp HB, Traystman RJ, Macleod MR (2010) Different strokes for different folks: the rich diversity of animal models of focal cerebral ischemia. J Cerebr Blood F Met 30(8):1412-1431

12. Rice JE, Vannucci RC, Brierley JB (1981) The influence of immaturity on hypoxic-ischemic brain damage in the rat. Ann Neurol 9(2):131-141

13. Sicard K, Fisher M (2009) Animal models of focal brain ischemia. Exp Transl Stroke Med 1(1):7

14. Sternlicht MD, Werb Z (2001) How matrix metalloproteinases regulate cell behavior. Ann Rev Cell Dev Biol 17:463
15. Dzwonek J, Rylski M, Kaczmarek L (2004) Matrix metalloproteinases and their endogenous inhibitors in neuronal physiology of the adult brain. FEBS Lett 567(1):129-135

16. Rivera S, Khrestchatisky M, Kaczmarek L, Rosenberg GA, Jaworski DM (2010) Metzincin proteases and their inhibitors: foes or friends in nervous system physiology? J Neurosci 30(46):1533715357

17. Huntley GW (2012) Synaptic circuit remodelling by matrix metalloproteinases in health and disease. Nat Rev Neurosci 13(11):743-757

18. Kaczmarek L (2012) MMP-9 inhibitors in the brain: can old bullets shoot new targets? Curr Pharm Des 19(6):1085-1089

19. Rybakowski JK (2009) Matrix metalloproteinase-9 (MMP-9): a mediating enzyme in cardiovascular disease, cancer, and neuropsychiatric disorders. Cardiovascular psychiatry and neurology 2009 (09): Article ID 904836

20. Lakhan SE, Kirchgessner A, Tepper D, Leonard A (2013) Matrix metalloproteinases and blood-brain barrier disruption in acute ischemic stroke. Front Neurol 4

21. George SJ, Johnson JL (2010) In situ zymography. In: Matrix Metalloproteinase Protocols. Springer, pp 271-277

22. Gawlak M, Górkiewicz T, Gorlewicz A, Konopacki FA, Kaczmarek L, Wilczynski GM (2009) High resolution in situ zymography reveals matrix metalloproteinase activity at glutamatergic synapses. Neuroscience 158(1):167-176

23. Castellanos M, Leira R, Serena J, Pumar JM, Lizasoain I, Castillo J, Davalos A (2003) Plasma metalloproteinase-9 concentration predicts hemorrhagic transformation in acute ischemic stroke. Stroke 34(1):40-46

24. Montaner J, Molina CA, Monasterio J, Abilleira S, Arenillas JF, Ribo M, Quintana M, Alvarez-Sabin J (2003) Matrix metalloproteinase-9 pretreatment level predicts intracranial hemorrhagic complications after thrombolysis in human stroke. Circulation 107(4):598-603

25. Horstmann S, Kalb P, Koziol J, Gardner H, Wagner S (2003) Profiles of matrix metalloproteinases, their inhibitors, and laminin in stroke patients: influence of different therapies. Stroke 34(9): 2165-2170

26. Heo JH, Kim SH, Lee KY, Kim EH, Chu CK, Nam JM (2003) Increase in plasma matrix metalloproteinase- 9 in acute stroke patients with thrombolysis failure. Stroke 34(6):48-50

27. Reynolds MA, Kirchick HJ, Dahlen JR, Anderberg JM, McPherson PH, Nakamura KK, Laskowitz DT, Valkirs GE, Buechler KF (2003) Early biomarkers of stroke. Clin Chem 49(10):1733-1739

28. Rosenberg GA, Cunningham LA, Wallace J, Alexander S, Estrada EY, Grossetete M, Razhagi A, Miller K, Gearing A (2001) Immunohistochemistry of matrix metalloproteinases in reperfusion injury to rat brain: activation of MMP-9 linked to stromelysin-1 and microglia in cell cultures. Brain Res 893(1-2):104-112

29. Planas AM, Sole S, Justicia C (2001) Expression and activation of matrix metalloproteinase-2 and -9 in rat brain after transient focal cerebral ischemia. Neurobiol Dis 8(5):834-846

30. Rosell A, Ortega-Aznar A, Alvarez-Sabin J, Fernandez-Cadenas I, Ribo M, Molina CA, Lo EH, Montaner J (2006) Increased brain expression of matrix metalloproteinase- 9 after ischemic and hemorrhagic human stroke. Stroke 37(6):1399-1406

31. Rivera S, Ogier C, Jourquin J, Timsit S, Szklarczyk AW, Miller K, Gearing AJ, Kaczmarek L, Khrestchatisky M (2002) Gelatinase B and TIMP-1 are regulated in a cell- and time-dependent manner in association with neuronal death and glial reactivity after global forebrain ischemia. Eur J Neurosci 15(1):19-32

32. Rosell A, Lo EH (2008) Multiphasic roles for matrix metalloproteinases after stroke. Curr Opin Pharmacol 8(1):82-89

33. Lakhan SE, Kirchgessner A, Hofer M (2009) Inflammatory mechanisms in ischemic stroke: therapeutic approaches. J Transl Med 7(1):97

34. Yamashita T, Abe K (2011) Therapeutic approaches to vascular protection in ischemic stroke. Acta Med Okayama 65:219-223 
35. Fagan SC, Hess DC, Hohnadel EJ, Pollock DM, Ergul A (2004) Targets for vascular protection after acute ischemic stroke. Stroke 35(9):2220-2225

36. Lee SR, Tsuji K, Lo EH (2004) Role of matrix metalloproteinases in delayed neuronal damage after transient global cerebral ischemia. J Neurosci 24(3):671-678

37. Copin JC, Goodyear MC, Gidday JM, Shah AR, Gascon E, Dayer A, Morel DM, Gasche Y (2005) Role of matrix metalloproteinases in apoptosis after transient focal cerebral ischemia in rats and mice. Eur J Neurosci 22(7):1597-1608

38. Lee SR, Lo EH (2004) Induction of caspase-mediated cell death by matrix metalloproteinases in cerebral endothelial cells after hypoxia-reoxygenation. J Cereb Blood F Met 24(7):720-727

39. Kelly PJ, Morrow JD, Ning M, Koroshetz W, Lo EH, Terry E, Milne GL, Hubbard J, Lee H, Stevenson E, Lederer M, Furie KL (2008) Oxidative stress and matrix metalloproteinase-9 in acute ischemic stroke: the Biomarker Evaluation for Antioxidant Therapies in Stroke (BEAT-Stroke) study. Stroke 39(1):100-104

40. Yang Y, Candelario-Jalil E, Thompson JF, Cuadrado E, Estrada EY, Rosell A, Montaner J, Rosenberg GA (2010) Increased intranuclear matrix metalloproteinase activity in neurons interferes with oxidative DNA repair in focal cerebral ischemia. J Neurochem 112(1): 134-149

41. Zhao BQ, Wang S, Kim HY, Storrie H, Rosen BR, Mooney DJ, Wang X, Lo EH (2006) Role of matrix metalloproteinases in delayed cortical responses after stroke. Nat Med 12(4):441-445

42. Inzitari D, Giusti B, Nencini P, Gori AM, Nesi M, Palumbo V, Piccardi B, Armillis A, Pracucci G, Bono G (2013) MMP-9 variation after thrombolysis is associated with hemorrhagic transformation of lesion and death. Stroke

43. Ning M, Furie KL, Koroshetz WJ, Lee H, Barron M, Lederer M, Wang X, Zhu M, Sorensen AG, Lo EH, Kelly PJ (2006) Association between $\mathrm{PA}$ therapy and raised early matrix metalloproteinase-9 in acute stroke. 66(10):1550-1555

44. Sumii T, Lo EH (2002) Involvement of matrix metalloproteinase in thrombolysis-associated hemorrhagic transformation after embolic focal ischemia in rats. Stroke 33(3):831-836

45. Yang DY, Pan HC, Chen CJ, Cheng FC, Wang YC (2007) Effects of tissue plasminogen activator on cerebral microvessels of rats during focal cerebral ischemia and reperfusion. Neurol Res 29(3):274-282

46. Zhao BQ, Ikeda Y, Ihara H, Urano T, Fan W, Mikawa S, Suzuki Y, Kondo K, Sato K, Nagai N, Umemura K (2004) Essential role of endogenous tissue plasminogen activator through matrix metalloproteinase 9 induction and expression on heparin-produced cerebral hemorrhage after cerebral ischemia in mice. Blood 103(7):2610 2616

47. Kelly MA, Shuaib A, Todd KG (2006) Matrix metalloproteinase activation and blood-brain barrier breakdown following thrombolysis. Exp Neurol 200(1):38-49

48. Clark AW, Krekoski CA, Bou SS, Chapman KR, Edwards DR (1997) Increased gelatinase A (MMP-2) and gelatinase B (MMP9) activities in human brain after focal ischemia. Neurosci Lett 238(1-2):53-56

49. Heo JH, Lucero J, Abumiya T, Koziol JA, Copeland BR, del Zoppo GJ (1999) Matrix metalloproteinases increase very early during experimental focal cerebral ischemia. J Cereb Blood Flow Metab 19(6):624-633

50. Gasche Y, Fujimura M, Morita-Fujimura Y, Copin JC, Kawase M, Massengale J, Chan PH (1999) Early appearance of activated matrix metalloproteinase- 9 after focal cerebral ischemia in mice: a possible role in blood-brain barrier dysfunction. J Cereb Blood Flow Metab 19(9):1020-1028

51. Fujimura M, Gasche Y, Morita-Fujimura Y, Massengale J, Kawase M, Chan PH (1999) Early appearance of activated matrix metalloproteinase- 9 and blood-brain barrier disruption in mice after focal cerebral ischemia and reperfusion. Brain Res 842(1):92-100
52. Maddahi A, Chen Q, Edvinsson L (2009) Enhanced cerebrovascular expression of matrix metalloproteinase- 9 and tissue inhibitor of metalloproteinase-1 via the MEK/ERK pathway during cerebral ischemia in the rat. BMC Neurosci 10:56

53. Rosell A, Ortega-Aznar A, Alvarez-Sabín J, Fernández-Cadenas I, Ribó M, Molina CA, Lo EH, Montaner J (2006) Increased brain expression of matrix metalloproteinase- 9 after ischemic and hemorrhagic human stroke. Stroke 37(6):1399-1406

54. Amantea D, Corasaniti MT, Mercuri NB, Bernardi G, Bagetta G (2008) Brain regional and cellular localization of gelatinase activity in rat that have undergone transient middle cerebral artery occlusion. Neuroscience 152(1):8-17

55. Morancho A, Rosell A, Garcia-Bonilla L, Montaner J (2010) Metalloproteinase and stroke infarct size: role for antiinflammatory treatment? Ann N Y Acad Sci 1207:123-133

56. Ramos-Fernandez M, Bellolio MF, Stead LG (2011) Matrix metalloproteinase-9 as a marker for acute ischemic stroke: a systematic review. J Stroke Cerebrovasc Dis 20(1):47-54

57. Wang X, Jung J, Asahi M, Chwang W, Russo L, Moskowitz MA, Dixon CE, Fini ME, Lo EH (2000) Effects of matrix metalloproteinase- 9 gene knock-out on morphological and motor outcomes after traumatic brain injury. J Neurosci 20(18):7037-7042

58. Asahi M, Wang X, Mori T, Sumii T, Jung J-C, Moskowitz MA, Fini ME, Lo EH (2001) Effects of matrix metalloproteinase-9 gene knock-out on the proteolysis of blood-brain barrier and white matter components after cerebral ischemia. J Neurosci 21(19):7724-7732

59. Svedin P, Hagberg H, Savman K, Zhu C, Mallard C (2007) Matrix metalloproteinase-9 gene knock-out protects the immature brain after cerebral hypoxia-ischemia. J Neurosci 27(7):1511-1518

60. Ludewig P, Sedlacik J, Gelderblom M, Bernreuther C, Korkusuz Y, Wagener C, Gerloff C, Fiehler J, Magnus T, Horst AK (2013) CEACAM1 Inhibits MMP-9-mediated blood-brain-barrier breakdown in a mouse model for ischemic stroke. Circ Res PMID: 23780386:[Epub ahead of print]

61. Rivera S, Tremblay E, Timsit S, Canals O, Ben-Ari Y, Khrestchatisky M (1997) Tissue inhibitor of metalloproteinases-1 (TIMP-1) is differentially induced in neurons and astrocytes after seizures: evidence for developmental, immediate early gene, and lesion response. J Neurosci 17(11):4223-4235

62. Tejima E, Guo S, Murata Y, Arai K, Lok J, van Leyen K, Rosell A, Wang X, Lo EH (2009) Neuroprotective effects of overexpressing tissue inhibitor of metalloproteinase TIMP-1. J Neurotrauma 26(11): 1935-1941

63. Fujimoto M, Takagi Y, Aoki T, Hayase M, Marumo T, Gomi M, Nishimura M, Kataoka H, Hashimoto N, Nozaki K (2008) Tissue inhibitor of metalloproteinases protect blood-brain barrier disruption in focal cerebral ischemia. J Cereb Blood Flow Metab 28(10):1674-1685

64. Cheng YD, Al-Khoury L, Zivin JA (2004) Neuroprotection for ischemic stroke: two decades of success and failure. NeuroRx 1(1):36- 45

65. Dong X, Song Y-N, Liu W-G, Guo X-L (2009) Mmp-9, a potential target for cerebral ischemic treatment. Curr Neuropharmacol 7(4):269

66. Hu Q, Chen C, Khatibi NH, Li L, Yang L, Wang K, Han J, Duan W, Zhang JH, Zhou C (2011) Lentivirus-mediated transfer of MMP-9 shRNA provides neuroprotection following focal ischemic brain injury in rats. Brain Res 1367:347-359

67. Souvenir R, Fathali N, Ostrowski RP, Lekic T, Zhang JH, Tang J (2011) Tissue inhibitor of matrix metalloproteinase-1 mediates erythropoietin-induced neuroprotection in hypoxia ischemia. Neurobiol Dis 44(1):28-37

68. Tan HK, Heywood D, Ralph GS, Bienemann A, Baker AH, Uney JB (2003) Tissue inhibitor of metalloproteinase 1 inhibits excitotoxic cell death in neurons. Mol Cell Neurosci 22(1):98-106

69. Chaturvedi M, Figiel I, Sreedhar B, Kaczmarek L (2012) Neuroprotection from tissue inhibitor of metalloproteinase-1 and its nanoparticles. Neurochem Int 61(7):1065-1071 
70. Romanic AM, White RF, Arleth AJ, Ohlstein EH, Barone FC (1998) Matrix metalloproteinase expression increases after cerebral focal ischemia in rats inhibition of matrix metalloproteinase-9 reduces infarct size. Stroke 29(5):1020-1030

71. Hu Q, Chen C, Yan J, Yang X, Shi X, Zhao J, Lei J, Yang L, Wang K, Chen L (2009) Therapeutic application of gene silencing MMP-9 in a middle cerebral artery occlusion-induced focal ischemia rat model. Exp Neurol 216(1):35-46

72. Mahajan SD, Aalinkeel R, Reynolds JL, Nair B, Sykes DE, Bonoiu A, Roy I, Yong K-T, Law W-C, Bergey EJ (2012) Suppression of MMP-9 expression in brain microvascular endothelial cells (BMVEC) using a gold nanorod (GNR)-siRNA nanoplex. Immunol Invest 41(4):337-355

73. Bonoiu A, Mahajan SD, Ye L, Kumar R, Ding H, Yong KT, Roy I, Aalinkeel R, Nair B, Reynolds JL, Sykes DE, Imperiale MA, Bergey EJ, Schwartz SA, Prasad PN (2009) MMP-9 gene silencing by a quantum dot-siRNA nanoplex delivery to maintain the integrity of the blood brain barrier. Brain Res 1282:142-155

74. Magnoni S, Baker A, Thomson S, Jordan G, George S, McColl B, McCulloch J, Horsburgh K (2007) Neuroprotective effect of adenoviral-mediated gene transfer of TIMP-1 and -2 in ischemic brain injury. Gene Ther 14(7):621-625

75. Sa Y, Hao J, Samineni D, Clark J, Pyne-Geithman G, Broderick J, $\mathrm{Lu} \mathrm{A}$ (2011) Brain distribution and elimination of recombinant human TIMP-1 after cerebral ischemia and reperfusion in rats. Neurol Res 33(4):433-438

76. Batra J, Robinson J, Mehner C, Hockla A, Miller E, Radisky DC, Radisky ES (2012) PEGylation extends circulation half-life while preserving in vitro and in vivo activity of tissue inhibitor of metalloproteinases-1 (TIMP-1). PLoS One 7(11):e50028

77. Guan W, Kozak A, Fagan SC (2011) Drug repurposing for vascular protection after acute ischemic stroke. In: Intracerebral hemorrhage research. Springer, New York, pp 295-298

78. Hess DC, Fagan SC (2010) Repurposing an old drug to improve the use and safety of tissue plasminogen activator for acute ischemic stroke: minocycline. Pharmacotherapy: J Human Pharmacol Drug Ther 30(7P2):55S-61S

79. Sapadin AN, Fleischmajer R (2006) Tetracyclines: nonantibiotic properties and their clinical implications. J Am Acad Dermatol 54(2):258-265

80. Guerin C, Laterra J, Masnyk T, Golub LM, Brem H (1992) Selective endothelial growth inhibition by tetracyclines that inhibit collagenase. Biochem Biophys Res Commun 188(2):740-745

81. Machado LS, Kozak A, Ergul A, Hess DC, Borlongan CV, Fagan SC (2006) Delayed minocycline inhibits ischemia-activated matrix metalloproteinases 2 and 9 after experimental stroke. BMC Neurosci 7(1):56

82. Fagan SC, Cronic LE, Hess DC (2011) Minocycline development for acute ischemic stroke. Transl Stroke Res 2(2):202-208

83. Machado LS, Sazonova IY, Kozak A, Wiley DC, El-Remessy AB, Ergul A, Hess DC, Waller JL, Fagan SC (2009) Minocycline and tissue-type plasminogen activator for stroke assessment of interaction potential. Stroke 40(9):3028-3033

84. Zemke D, Majid A (2004) The potential of minocycline for neuroprotection in human neurologic disease. Clin Neuropharmacol 27(6):293-298

85. Chen X, Chen S, Jiang Y, Zhu C, Wu A, Ma X, Peng F, Ma L, Zhu D, Wang Q (2012) Minocycline reduces oxygen-glucose deprivationinduced PC12 cell cytotoxicity via matrix metalloproteinase-9, integrin $\beta 1$ and phosphorylated Akt modulation. Neurological Sciences: $1-6$

86. Koistinaho M, Malm TM, Kettunen MI, Goldsteins G, Starckx S, Kauppinen RA, Opdenakker G, Koistinaho J (2005) Minocycline protects against permanent cerebral ischemia in wild type but not in matrix metalloprotease-9-deficient mice. J Cereb Blood Flow Metab 25(4):460-467
87. Murata Y, Rosell A, Scannevin RH, Rhodes KJ, Wang X, Lo EH (2008) Extension of the thrombolytic time window with minocycline in experimental stroke. Stroke 39(12):3372-3377

88. Lee R (2011) Letter by Lee regarding article, "matrix metalloproteinase9 in an exploratory trial of intravenous minocycline for acute stroke". Stroke 42(10): e566-e566

89. Fan X, Lo EH, Wang X (2013) Effects of minocycline plus tissue plasminogen activator combination therapy after focal embolic stroke in type 1 diabetic rats. Stroke 44(3):745-752

90. Zhang L, Chopp M, Jia L, Cui Y, Lu M, Zhang ZG (2009) Atorvastatin extends the therapeutic window for tPA to $6 \mathrm{~h}$ after the onset of embolic stroke in rats. J Cereb Blood F Met 29(11):1816-1824

91. Jang J-W, Lee J-K, Lee M-C, Piao M-S, Kim S-H, Kim H-S (2012) Melatonin reduced the elevated matrix metalloproteinase-9 level in a rat photothrombotic stroke model. J Neurol Sci 323:221-227

92. Wu Y, Wang Y-P, Guo P, Ye X-H, Wang J, Yuan S-Y, Yao S-L, Shang Y (2012) A lipoxin A4 analog ameliorates blood-brain barrier dysfunction and reduces MMP-9 expression in a rat model of focal cerebral ischemia-reperfusion injury. J Mol Neurosci 46(3): 483-491

93. Zhou J, Li J, Rosenbaum DM, Barone FC (2010) Thrombopoietin protects the brain and improves sensorimotor functions: reduction of stroke-induced MMP-9 upregulation and blood-brain barrier injury. J Cereb Blood F Met 31(3):924-933

94. Hosomi N, Nishiyama A, Ban C, Naya T, Takahashi T, Kohno M, Koziol J (2005) Angiotensin type 1 receptor blockage improves ischemic injury following transient focal cerebral ischemia. Neuroscience 134(1):225-231

95. Candelario-Jalil E, Taheri S, Yang Y, Sood R, Grossetete M, Estrada EY, Fiebich BL, Rosenberg GA (2007) Cyclooxygenase inhibition limits blood-brain barrier disruption following intracerebral injection of tumor necrosis factor- $\alpha$ in the rat. J Pharmacol Exp Therapeut 323(2):488-498

96. Diener HC, Schneider D, Lampl Y, Bornstein NM, Kozak A, Rosenberg G (2008) DP-b99, a membrane-activated metal ion chelator, as neuroprotective therapy in ischemic stroke. Stroke 39(6): 1774-1778

97. Jiang X-F, Namura S, Nagata I (2001) Matrix metalloproteinase inhibitor KB-R7785 attenuates brain damage resulting from permanent focal cerebral ischemia in mice. Neurosci Lett 305(1):41-44

98. Gu Z, Cui J, Brown S, Fridman R, Mobashery S, Strongin AY, Lipton SA (2005) A highly specific inhibitor of matrix metalloproteinase-9 rescues laminin from proteolysis and neurons from apoptosis in transient focal cerebral ischemia. J Neurosci 25(27):6401-6408

99. Ranasinghe HS, Scheepens A, Sirimanne E, Mitchell MD, Williams CE, Fraser M (2012) Inhibition of MMP-9 activity following hypoxic ischemia in the developing brain using a highly specific inhibitor. Dev Neurosci 34(5):417-427

100. Coimbra C, Drake M, Boris-Möller F, Wieloch T (1996) Longlasting neuroprotective effect of postischemic hypothermia and treatment with an anti-inflammatory/antipyretic drug evidence for chronic encephalopathic processes following ischemia. Stroke 27(9):1578-1585

101. van der Worp HB, Macleod MR, Kollmar R (2010) Therapeutic hypothermia for acute ischemic stroke: ready to start large randomized trials\&quest. J Cereb Blood F Met 30(6):1079-1093

102. Wagner S, Nagel S, Kluge B, Schwab S, Heiland S, Koziol J, Gardner H, Hacke W (2003) Topographically graded postischemic presence of metalloproteinases is inhibited by hypothermia. Brain Res 984(1):63-75

103. Nagel S, Su Y, Horstmann S, Heiland S, Gardner H, Koziol J, Martinez-Torres FJ, Wagner S (2008) Minocycline and hypothermia for reperfusion injury after focal cerebral ischemia in the rat: effects on BBB breakdown and MMP expression in the acute and subacute phase. Brain Res 1188:198 
104. Lee JE, Yoon YJ, Moseley ME, Yenari MA (2005) Reduction in levels of matrix metalloproteinases and increased expression of tissue inhibitor of metalloproteinase- 2 in response to mild hypothermia therapy in experimental stroke. J Neurosurg 103(2):289-297

105. Truettner JS, Alonso OF, Dietrich WD (2005) Influence of therapeutic hypothermia on matrix metalloproteinase activity after traumatic brain injury in rats. J Cerebr Blood F Met 25(11):1505-1516

106. Zhao J-K, Guan F-L, Duan S-R, Zhao J-W, Sun R-H, Zhang L-M, Wang D-S (2013) Effect of focal mild hypothermia on expression of
MMP-9, TIMP-1, Tau-1 and $\beta$-APP in rats with cerebral ischaemia/ reperfusion injury. Brain Injury (0):1-9

107. Groysman LI, Emanuel BA, Kim-Tenser MA, Sung GY, Mack WJ (2011) Therapeutic hypothermia in acute ischemic stroke. Neurosurg Focus 30(6):E17

108. Takamiya M, Miyamoto Y, Yamashita T, Deguchi K, Ohta Y, Abe K (2012) Strong neuroprotection with a novel platinum nanoparticle against ischemic stroke-and tissue plasminogen activator-related brain damages in mice. Neuroscience 221:47-55 